\title{
Normal Fibronectin Levels as a Function of Age in the Pediatric Population
}

\author{
MICHAEL H. MCCAFFERTY ${ }^{(20)}$ MARTHA LEPOW, THOMAS M. SABA, ${ }^{(21)}$ ESHIN CHO, \\ HILAIRE MEUWISSEN, JOHN WHITE, AND SHARON F. ZUCKERBROD
}

Departments of Physiology and Pediatrics, Albany Medical College of Union University, Albany, New York, USA

\begin{abstract}
Summary
Fibronectin is an important non-immune opsonic protein influencing phagocytic clearance of blood-borne nonbacterial particulates which may arise in association with septic shock, tissue injury, and intravascular coagulation. In the present study, serum fibronectin was measured by both electroimmunoassay as well as rapid immunoturbidimetric assay in healthy children $(n=114)$ ranging in age from 1 month to 15 years in order to delineate the temporal alterations in fibronectin with age. Normal adult serum fibronectin concentrations are typically $220 \mu \mathrm{g} / \mathrm{ml} \pm 20 \mu \mathrm{g} / \mathrm{ml}$. Serum concentration is $35-40 \%$ lower than normal plasma concentration due to the binding of fibronectin to fibrin during clot formation. Children between 1-12 months of age had significantly $(P<0.05)$ lower serum fibronectin levels than children between the ages of 1-15 years. Progressive elevation in fibronectin levels was observed within the last 8 months of the first year of age. Fibronectin levels in children older than 1 year of age remained constant up to 15 years and were within the lower limit of the normal adult concentration. No significant $(P>0.05)$ difference in serum fibronectin was observed between male and female children at all age groups. Fibronectin levels thus, increase during the first year of age and normal levels of this blood protein in the infant are less than the normal range for adults.
\end{abstract}

Abbreviation

RES, reticuloendothelial system

Alterations in the serum concentration of immunoglobulin and complement as well as changes in phagocytic function are seen in children with increasing age. Fibronectin is a 440,000 dalton glycoprotein that circulates in adult males at a plasma concentration of $300-350 \mu \mathrm{g} / \mathrm{ml}$ with lower levels observed in adult females $(9,16,17)$. Serum levels are lower than plasma levels (14) due to the binding of fibronectin to fibrin during clot formation (9). Fibronectin has binding sites for collagen, fibrin, fibrinogen, actin and Staphylococcus aureus as previously reviewed $(9,13)$. Fibronectin serves as an opsonin to facilitate RES and leukocyte clearance of nonbacterial particulate material such as denatured collagen-coated particles, fibrin monomer, and injured cells $(1,2$, 13).

Fibronectin deficiency is seen acutely after trauma or burn and in association with sepsis in injured patients $(5,13,15,16)$. Deficiency also exists in critically-ill adult patients with disseminated intravascular coagulation and organ failure (10). Recent data suggest that fibronectin may also be a cofactor working in conjunction with complement for optimal binding and/or internalization of Staphylococcus aureus by neutrophils (6).

Much interest exists as to fibronectin levels in septic injured adult patients $(10,13,14,17)$, and the ability to reverse fibronectin deficiency by infusion of fresh plasma cryoprecipitate $(15,16)$. Although the range of normal has been defined by several studies in the adult population, changes in circulating fibronectin in the pediatric population with age has not been reported. This may be important in terms of appropriate interpretation of prevailing levels in children with various disease states, because normal concentrations in children may be different from normal levels in adults. This study was designed to measure fibronectin levels in a large group ( $n=114)$ of normal children in order to define its normal concentration as a function of age.

\section{MATERIALS AND METHODS}

Healthy children $(n=114)$ ranging in age from 1 month to 15 years were seen as outpatients or as inpatients for minor surgical procedures. No child studied had a history to suggest a recent infection or chronic disease. In the present study, in order to complete the large number of determinations in duplicate with parallel comparisons of two different assays, serum was collected for analysis because it could be frozen to allow for assay in groups at the same time. Fibronectin levels in serum are lower than plasma, but serum can be stored at $-16^{\circ} \mathrm{C}$ with confidence as to stability of the antigen for determinations by immunoassay $(2$, 14). Blood was allowed to clot for one hour at $25^{\circ} \mathrm{C}$. Thereafter, it was centifuged and the serum obtained was promptly frozen. For this particular study, the frozen samples were thawed as a group so that the samples could be assayed together with the same standards.

Fibronectin concentration was determined by both rocket electroimmunoassay (2) as well as the newly developed rapid immunoturbidimetric assay (14). Use of the new rapid immunoturbidimetric assay in the present investigation also allowed for further evaluation of its clinical utility with respect to ease of performance and rapidity (14). Samples were run in duplicate when using the immunoturbidimetric assay. For immunoturbidimetric assay, a self-contained kit with a range of calibrated human fibronectin standards and monospecific antiserum is available commercially (Biodynamics/BMC, Indianapolis, IN) as reported (14). For the electroimmunoassay, the fibronectin was isolated from normal human serum by affinity chromatography using gelatin-Sepharose, and antiserum was developed in rabbits and rendered monospecific as previously described $(1,2)$. The details for execution of both the electroimmunoassay as well as the immunoturbidimetric assay have been previously described in detail $(2,14,15$, 16). The levels observed were grouped by age using the following age groups: $1-4$ months $(n=14) ; 5-8$ months $(n=7)$; $9-12$ months $(n=11) ; 1-3$ years $(n=17) ; 3-5$ years $(n=19) ; 5-8$ years $(n=$ $21)$; $8-11$ years $(n=19)$; and $11-15$ years $(n=6)$. Statistical analysis for group differences relative to fibronectin concentration was performed by one-way analysis of variance on a DEC-10 Systems Computer.

\section{RESULTS}

Fibronectin levels as determined by either assay were significantly lower among the three groups of children less than one year of age than in older children (Figs. 1 and 2). Also, a rise in 


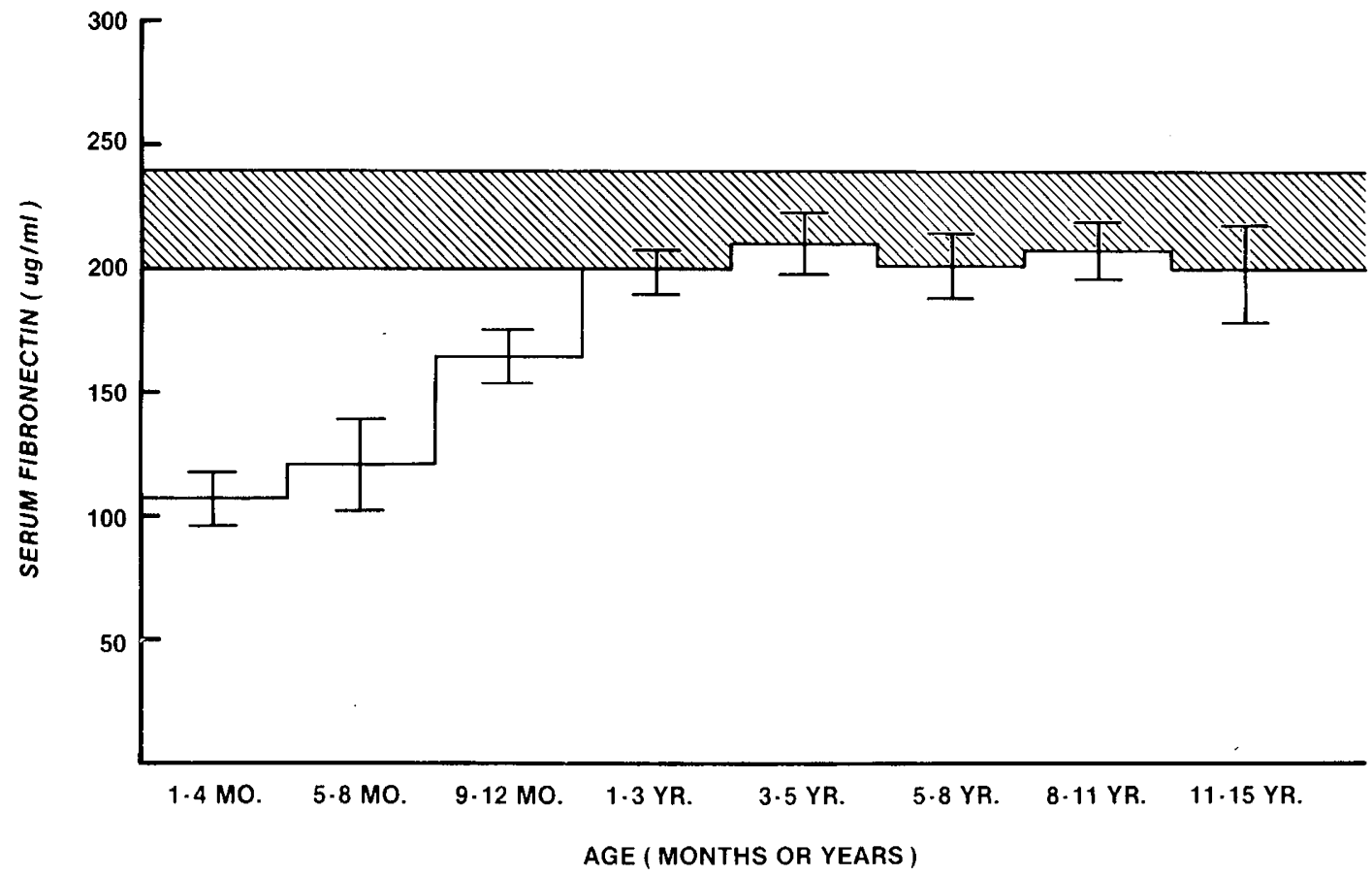

Fig. 1. Serum fibronectin as measured by rocket electroimmunoassay in children as a function of age. Shaded area presents the mean \pm S.E. of normal serum fibronectin levels observed in adults as determined by immunoturbidimetric assay. Each point reflects mean \pm S.E. The levels in the 14-month-, 5-8-month-, and 8-12-month-old group are significantly $(P<0.05)$ less than those in the 5-8-year-old group.

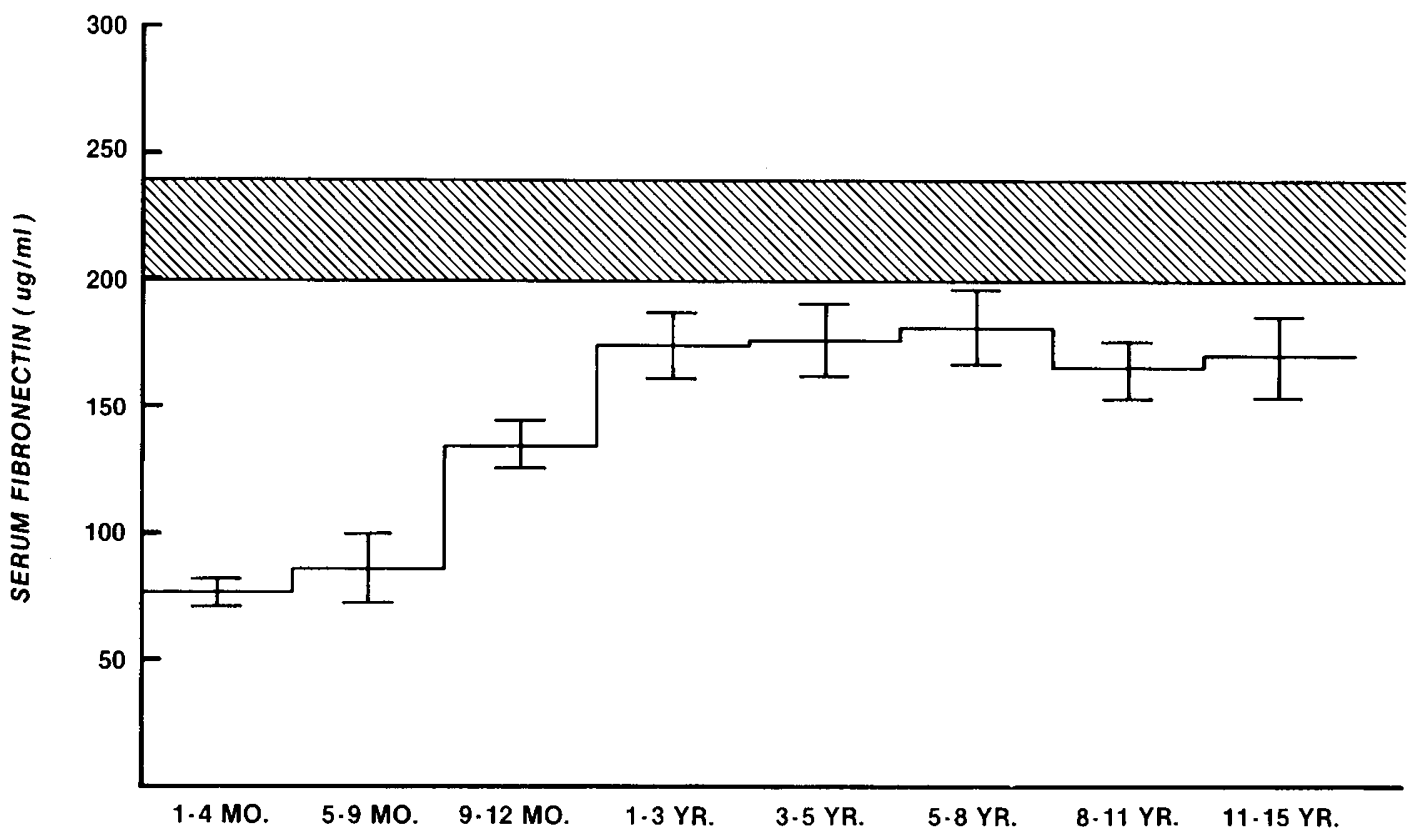

AGE ( MONTHS OR YEARS)

Fig. 2. Serum fibronectin as measured by immunoturbidimetric assay in children as a function of age. Shaded area presents the mean \pm S.E. of normal serum fibronectin levels observed in adults as determined by immunoturbidimetric assay. Each point reflects mean \pm S.E. The levels in the $1-$ 4-month-, 5-8-month-, and 8-12-month-old group are significantly $(P<0.05)$ less than those in the 5-8-year-old group.

fibronectin during the first year of life, especially between 5-12 months of age, was seen with both assay techniques. Fibronectin levels were not different from each other among the groups of children older than 1 year of age by either assay. If the 5-8-yearold group $(n=21)$, which was midway in the age profile studied, was used as representative of the larger group of children older than 1 year of age for statistical comparisons, it was observed that no differences existed between groups over the age interval of $1-$
15 years. In comparison to the 5-8-year-old group, children 1-4 months of age $(n=14)$ had fibronectin levels $43 \%$ and $53 \% ; 5-8$ month old children $(n=7)$ had levels $48 \%$ and 60\%; and 9-12 month old children $(n=11)$ had levels $75 \%$ and $82 \%$ of this reference group by immunoturbidimetric and electroimmunoassay, respectively (Figs. 1 and 2). As reflected in both assays, fibronectin concentrations of the older children approached the lower level of the normal adult levels (Figs. 1 and 2). The 
correlation coefficient between the electroimmunoassay and the immunoturbidimetric assay was $r=0.924$ and thus the agedependent alterations were apparent with either assay.

Previous determinations of fibronectin levels in adults document a lower fibronectin level in females as compared to males $(9,17)$. Whether this relationship also exists in children has not been determined. Accordingly, the children studied were further analyzed with respect to male $(n=78)$ and female $(n=36)$ group differences. No consistent difference in fibronectin levels was observed between male and female children at all of the eight age groupings identified in the previous two figures. Although not presented in the current paper, parallel studies in progress have been evaluating the fibronectin levels in neonates grouped according to birth weight. Low but definable concentrations (about 25$35 \%$ of adult) exist in $1000-1500 \mathrm{~g}$ infants with a progressive elevation in the average fibronectin concentration up to the weight of 2500-3000 g, at which time a plateau exists (Meuwissen, Saba, McCafferty, et al., unpublished findings).

\section{DISCUSSION}

Although the clinical evaluation of the immunoturbidimetric assay was not the primary focus of this study, the data obtained did demonstrate the utility of the rapid immunoturbidimetric assay for fibronectin. Inasmuch as both assays require a small serum aliquot for the determination, Laurell rocket electroimmunoassay requires $24-48 \mathrm{~h}$ for obtaining results, whereas the immunoturbidimetric assay provides quantitation in $10 \mathrm{~min}$ (14). The temporal pattern was the same with both assays, although the absolute values were slightly but consistently higher at all time intervals with the electroimmunoassay. This may be due to the different sources of the antibody used in the present study in the electroimmunoassay versus the immunoturbidimetric aassay. For the electroimmunoassay, the antisera was prepared in rabbits immunized by our laboratory $(15,16)$ with affinity purified human fibronectin. For the immunoturbidimetric assay, the antisera was prepared in sheep and already available in the commercial kits (14). The normal adult range identified in serum was determined by immunoturbidimetric assay.

Alterations of cellular and humoral immunity relative to adults exists in neonates. For example, adult levels of humoral mediators of host resistance are reached at varying ages with normal adult levels of IgA not being reached until puberty. The phagocytic capability of the RES in children with respect to clearance of test particle from the circulation is not well characterized. In the present study, circulating fibronectin levels in children over the age interval of 1 month to 1 year are very low relative to those concentrations reported in adults. Furthermore, a rapid and unexpected increase occurs during this age range such that children of 12 months of age or older have serum fibronectin levels within the lower level of normal adults. It was of interest that no consistent sex difference was observed among any of the age groups of children. This is in direct contrast to previous observations in adults where males have higher fibronectin levels than females (17). These observations suggest that hormonal influences may account for the adult differences. Accordingly, measurement of fibronectin levels among males and females before, during, and after puberty may be valuable to confirm and extend this hypothesis.

In animal studies, RES function in vivo is correlated with fibronectin levels and associated opsonic activity $(2,4,13)$. That the RES functional clearance capacity in vivo could parallel these fibronectin levels in patients can only be inferred. It is of interest, however, that previous determinations of bioassayable opsonic activity as related to opsonic protein (fibronectin; cold-insoluble globulin) have shown that newborn infants between the ages of 19 days have lower opsonic activity in serum as compared to adults when tested by the standard liver slice bioassay using the gelatincoated test particles (3).

Temporal changes in the ability of the RES to clear ingested test colloids in relationship to opsonic activity levels have been measured in neonatal rats as a function of age (8). In the neonatal period of the rat, bioassayable as well as immunoreactive fibronectin is lower than that observed in adult rats. But, hepatic Kupffer cell phagocytosis in terms of intrinsic cellular activity can at times be disproportionally activated $(4,8)$. As the rat matures, hepatic Kupffer cell function decreases and becomes more synchronized at 21-23 days with fibronectin levels and/or opsonic activity. In the rat, immunoreactive fibronectin attains normal levels at about 28-32 days of age (Lorenzen and Saba, unpublished data). These animal observations emphasize that RES function is a composite behavior influenced by humoral factors such as fibronectin and intrinsic cellular function.

Depression of RES phagocytic function has been correlated with decreased resistance to experimental shock and burn injury (13). Fibronectin deficiency induced by injection of specific antiserum to fibronectin or by injection of gelatinized particles also decreases resistance to experimental trauma and Staphylococcus aureus peritonitis $(7,13)$. Conversely, opsonic deficiency is seen after trauma and the degree of fibronectin deficiency appears to be directly $(5,12,13,16)$ correlated with the extent of trauma. Indeed, in septic-injured patients, persistent fibronectin deficiency is associated with development of adult respiratory distress characterized by a ventilation-perfusion imbalance, whereas infusion of fibronectin-rich cryoprecipitate appears to improve pulmonary function in preliminary clinical studies $(13,15,16)$. Although most such clinical studies have been done in adults, the recent documentation by Robbins, et al. (12) of utilization of cryoprecipitate infusion to reverse fibronectin (cold-insoluble globulin) deficiency in a 6-wk-old septic child emphasizes the need to document the normal temporal pattern of fibronectin with age in children. This may have direct clinical significance because fibronectin deficiency may influence the development of organ failure in septicinjured patients $(12,13,15)$ or patients with intravascular coagulation (10).

The discovery that opsonic glycoprotein was identical to coldinsoluble globulin or plasma fibronectin $(1,13)$, which is concentrated in cryoprecipitate, allows replacement therapy to be accomplished in patients (15). Preliminary clinical $(13,15,16)$ observations in septic surgical, trauma, and burn patients with cryoprecipitate are encouraging, but further controlled studies must be done to evaluate this potential modality of therapy. Indeed, any such therapy in children must be carefully viewed in the context of the normal developmental changes in circulating fibronectin levels. For example, serum concentrations of $100 \mu \mathrm{g} / \mathrm{ml}$ may be quite normal in a 6-month-old child, whereas such a level would reflect a significant deficiency in adults $(13,15,16)$.

Because circulating fibronectin appears to be an important modulator of phagocytic function and host defense against injury $(13,16)$, one might speculate that the ability of the 1-month- to 1year-old child to withstand a traumatic insult may be influenced by the low prevailing fibronectin level; however, the relationship of fibronectin to bacterial resistance is not clear because conflicting data exist as to whether it promotes Staphylococcus aureus ingestion by neutrophils (6) or whether it only promotes bacterial aggregation and/or binding to neutrophils (11). It is suggested that alterations in fibronectin that may develop during septic surgical complications in children warrants investigation. Furthermore, definition of the fibronectin level in premature newborns and newborns with respiratory distress may provide valuable insight as to the possible functional relationship between fibronectin deficiency and abnormal lung function in view of such a correlation in septic injured adults (13). The possibility that the relationship between fibronectin and lung vascular behavior is mediated by disturbances in the tissue fibronectin pool and not the circulating plasma fibronectin as speculated (13) need consideration.

\section{REFERENCES AND NOTES}

1. Blumenstock, F. A., Saba, T. M., Weber, P., and Laffin, R.: Biochemical and immunological characterization of human opsonic $\alpha_{2}$ SB glycoprotein: its identity with cold insoluble globulin. J. Biol. Chem., 253: 4287 (1978). 
2. Blumenstock, F. A., Weber, P., Saba, T. M., and Laffin, R.: Electroimmunoassay of alpha-2-opsonic protein during reticuloendothelial blockade. Am. J. Physiol., 232: R80 (1977)

3. Graham, C. W., Saba, T. M., Lolekha, S., and Gotoff, S.: Deficient serum opsonic activity for macrophage function in newborn infants. Proc. Soc. Exp Biol. Med., 143: 991 (1973).

4. Graham, C. W. and Saba, T. M.: Development of humoral and cellular aspects of macrophage function in fostered and nonfostered neonates. J. Reticul. Soc., 13: 7 (1973).

5. Lanser, M. E., Saba, T. M., and Scovill, W. A.: Opsonic glycoprotein (plasma fibronectin) levels after burn injury: relationship to extent of burn and development of sepsis. Ann. Surg., 192: 776 (1980).

6. Lanser, M. E. and Saba, T. M.: Fibronectin as a co-factor necessary for optima granulocyte phagocytosis of Staphylococcus aureus. J. Reticul. Soc., 30: 415 (1981).

7. Lanser, M. E. and Saba, T. M.: Opsonic fibronectin deficiency and sepsis: cause or effect? Ann. Surg., 195: 340 (1982).

8. Lorenzen, J. R. and Saba, T. M.: Reticuloendothelial system clearance capacity during neonatal development. Dev. Comp. Immunol., 3: 147 (1979)

9. Mosher, D. F.: Fibronectin. Prog. Hemostasis Thromb., 5: 111 (1980).

10. Mosher, D. F. and Williams, E. M.: Fibronectin concentration is decreased in plasma of severely ill patients with disseminated intravascular coagulation. $\mathbf{J}$ Lab. Clin. Med., 91: 729 (1978).

11. Proctor, R. A., Prendergast, E., and Mosher, D. F.: Fibronectin mediates attach ment of Staphylococcus aureus to human neutrophils. Blood, 59: 681 (1982)

12. Robbins, A. B., Doran, J. E., Reese, A. C., and Mansberger, A. R.: Cold-insoluble globulin levels in operative trauma: serum depletion, wound sequestration, and biological activity. Am. Surg., 46: 663, 1980.
13. Saba, T. M. and Jaffe, E.: Plasma fibronectin (opsonic glycoprotein): Its synthesis by vascular endothelial cells and role in cardiopulmonary integrity after trauma as related to reticuloendothelial function. Am. J. Med., 68: 577 (1980).

14. Saba, T. M., Alpert, W., Blumenstock, F. A., Evanega, G., Staehler, F., and Cho, E.: Evaluation of a rapid immunoturbidimetric assay for opsonic plasma fibronectin in surgical and trauma patients. J. Lab. Clin. Med., 98: 482 (1981).

15. Saba, T. M., Blumenstock, F. A., Scovill, W. A., and Bernard, H.: Cryoprecipitate reversal of opsonic $\alpha_{2}$-surface binding glycoprotein deficiency in septic surgical and trauma patients. Science, 201: 622 (1978).

16. Scovill, W. A Annest, S. J, Saba, T. M. Blumenstock, F, A Newell, J. C. Stratton, H. H., and Powers, S. R.: Cardiovascular hemodynamics after opsonic alpha-2-surface binding glycoprotein therapy in injured patients. Surgery, 86 : 284 (1979).

17. Stathakis, N. E., Tsianas, E., and Fountis, A.: Plasma fibronectin in normal subjects and in various disease states. J. Clin. Pathol., 34: 504 (1981).

18. This study was supported in part by GM 21447 from the Institute of General Medical Sciences and in part by the Clinical Studies Center grant M01-RR00749 from the National Institutes of Health.

19. The authors acknowledge the secretarial assistance of Syliva Deskewicz, Deborah Moran, and Maureen Davis.

20. Michael H. McCafferty, M.D. was an NIH Postdoctoral Research Fellow during the period of these studies and supported by GM-07033 entitled "Basic and Clinical Science Trauma Research Training."

21. Requests for reprints should be addressed to: Dr. Thomas M. Saba, Professor and Chairman, Department of Physiology, Albany Medical College, 47 New Scotland Avenue, Albany, New York 12208.

22. Received for publication June 9, 1982.

23. Accepted for publication October 12, 1982 